\title{
La circulation transatlantique d'un ethos scientifique pour la sociologie
}

La correspondance de Raymond Boudon

The transatlantic circulation of a scientific ethos for sociology : Raymond Boudon's letters

Michel Dubois et Sylvie Mesure

\section{OpenEdition}

\section{Journals}

Édition électronique

URL : https://journals.openedition.org/ress/4228

DOI : $10.4000 /$ ress. 4228

ISSN : 1663-4446

\section{Éditeur}

Librairie Droz

\section{Édition imprimée}

Date de publication : 14 décembre 2018

Pagination : 41-63

ISSN : 0048-8046

\section{Référence électronique}

Michel Dubois et Sylvie Mesure, «La circulation transatlantique d'un ethos scientifique pour la sociologie ", Revue européenne des sciences sociales [En ligne], 56-2 | 2018, mis en ligne le 14 décembre 2021, consulté le 08 janvier 2022. URL : http://journals.openedition.org/ress/4228 ; DOI : https:// doi.org/10.4000/ress. 4228 


\title{
LA CIRCULATION TRANSATLANTIQUE D'UN ETHOS SCIENTIFIQUE POUR LA SOCIOLOGIE
}

LA CORRESPONDANCE DE RAYMOND BOUDON

MICHEL DUBOIS \& SYLVIE MESURE

EpiDaPo - CNRS, George Washington University /

GEMASS - CNRS, Paris, Sorbonne Université michel.dubois@cnrs.fr/mesure.sylvie@wanadoo.fr

\begin{abstract}
Résumé. La circulation internationale des idées sociologiques entre la France et les États-Unis est une question qui intéresse traditionnellement autant les historiens des sciences sociales que les sociologues eux-mêmes. Cet article étudie les modalités de cette circulation transatlantique à partir du cas spécifique de Raymond Boudon. II exploite les documents récemment versés aux Archives nationales, en particulier la correspondance entre Boudon et deux figures centrales de la sociologie aux États-Unis: Paul F. Lazarsfeld et Robert K. Merton. Le dialogue, amorcé dès le début des années 1960, entre Boudon et les patrons du département de sociologie de l'Université Columbia à New York demeurera intense et vivant pendant de nombreuses années. L'article montre notamment de quelle manière Lazarsfeld et Merton ont contribué à accroître la circulation internationale des travaux de Boudon, et réciproquement de quelle manière ce dernier a tenté à sa manière de porter en France les qualités de l'ethos scientifique pour la sociologie incarné à ses yeux par le duo de Columbia.
\end{abstract}

Mots-clés: correspondance, ethos, internationalisation, Paul F. Lazarsfeld, réseau personnel, Raymond Boudon, Robert K. Merton.

\begin{abstract}
The international circulation of sociological ideas between France and the United States is an issue that has traditionally interested both historians and sociologists. This article studies the modalities of this transatlantic circulation, drawing on the specific case of French sociologist Raymond Boudon. It uses archives that have recently been made available, particularly the correspondence between Boudon and two central US sociological figures: Paul F. Lazarsfeld and Robert K. Merton. The dialogue, initiated in the early 1960s, between Boudon and the two "leading figures" of Columbia's Sociology Department, remained intense and lively for many years. The article shows how both Lazarsfeld and Merton contributed to increasing the international circulation of Boudon's work, and, reciprocally, how Boudon tried to import into France the qualities of the scientific ethos for sociology developed by the sociological duo from Columbia.
\end{abstract}

Keywords: correspondence, ethos, internationalization, Paul F. Lazarsfeld, personal network, Raymond Boudon, Robert K. Merton. 
La circulation internationale des idées sociologiques entre la France et les États-Unis est une question qui intéresse traditionnellement autant les historiens des sciences sociales que les sociologues eux-mêmes. Les approches disponibles sont multiples. Certaines sont centrées sur l'étude détaillée de «trajectoires» singulières: les voyages aux États-Unis, par exemple, de Maurice Halbwachs (Topalov, 2005), de Jean Stœetzel (Stankiewicz, 2008) ou de Michel Crozier (Chaubet, 20I3), etc. D’autres au contraire adoptent une perspective plus globalisante et discutent les conditions sociales et politiques de cette circulation et ses effets plus ou moins structurels (Pollak, 1976; Heilbron, 2005 ; Boncourt, 2016).

L'étude croisée de la réception des hommes et de leurs idées - des États-Unis vers la France (Marcel, 2004), ou inversement de la France vers les États-Unis (Ollion et Abbott, 20ı6), souligne fréquemment deux faits d'importance. D’une part, l'extrême inégalité des sociologues au regard de leur capacité à dépasser durablement les frontières nationales: une petite minorité parvient à capter et maintenir l'attention de leurs collègues étrangers, là où la grande majorité reste purement et simplement invisible et ignorée. Et cette invisibilité n’est pas toujours celle à laquelle on s'attend. D’autre part, le rôle central joué par un petit nombre de «médiateurs » qui, implantés dans le milieu académique national, jouent un rôle relationnel crucial à travers l'obtention d'invitations de séjours de recherche, de traductions et adaptations d'ouvrages ou de participations à des conférences.

Cet article contribue à l'étude de cette circulation internationale des sociologues à partir du cas spécifique de Raymond Boudon. La trajectoire sociologique de Boudon est intéressante du point de vue des rapports entre les communautés sociologiques française et étatsunienne pour au moins trois raisons. Tout d’abord parce que, comme l'a rappelé une étude récente ${ }^{2}$, Raymond Boudon fait partie des sociologues français les plus cités dans les revues américaines de sociologie (Ollion et Abbott, 20ı6). Ensuite, parce que la constitution de dépôts récents d’ar-

I Les auteurs remercient vivement Madame Rosemarie Boudon qui leur a accordé une totale liberté pour exploiter les archives de Raymond Boudon. Ils remercient également Brigitte Mazon qui a dirigé le travail d'archivage du fonds Boudon à l'École des hautes études en sciences sociales.

2 L'étude citationnelle réalisée par Étienne Ollion et Andrew Abbott (2016) sur la période 1970-2012 place Raymond Boudon en 6e position, derrière Émile Durkheim, Pierre Bourdieu, Bruno Latour, Alexis de Tocqueville et Michel Callon. 
chives $^{3}$ permet d'aborder cette trajectoire internationale à partir d'un matériau original qui souligne l'importance de deux figures classiques de la sociologie aux États-Unis que sont Paul F. Lazarsfeld et Robert K. Merton. Ces deux sociologues ont joué un rôle central pour le département de sociologie de l'université de Columbia, et plus largement pour la formation d'une conception scientifique de la sociologie durant la période d'après Seconde Guerre mondiale. Enfin, parce que la circulation de Boudon entre la France et les États-Unis a d'ores et déjà donné lieu à différents commentaires qu'il est possible de mettre à l'épreuve de ces archives. On se souvient notamment de la manière dont Boudon avait sèchement critiqué la reconstitution historique de son voyage à Columbia proposée par Henri Mendras dans ses Souvenirs d'un vieux mandarin (Mendras, 1995)

L'article est structuré en trois parties principales. Il revient dans un premier temps sur la nature des documents récemment versés aux Archives nationales. Il s’agit de préciser la diversité de ce matériau mais également la manière dont les auteurs de cet article ont entrepris d'en étudier, à partir de l'automne 20I4, une partie limitée: la correspondance générale de Boudon. Cette première section est notamment l'occasion de souligner la spécificité de cette correspondance, et de caractériser à gros traits la nature du réseau personnel informel autour duquel s'est organisée l’activité scientifique de Boudon des années 1960 jusqu’au début des années 2000. La deuxième partie de l'article recentre l'attention sur un sous-ensemble de la correspondance générale de Boudon, celle qu'il a entretenue avec Lazarsfeld et Merton. Elle présente les caractéristiques du matériau disponible mais également la manière dont ont été décrites la nature et l'importance de cette relation entre les départements de sociologie des universités de la Sorbonne et de Columbia. Enfin, la troisième partie analyse la

3 Ce cas n'est bien sûr pas inédit. Des dépôts récents d'archives de chercheurs sont autant d'incitations à développer des perspectives de recherche originale sur le développement de la sociologie en France (Borzeix et Rot, 2010) comme aux États-Unis (Dubois, 2014).

4 «Le livre contient en outre des erreurs de fait et des à-peu-près qui en font une source peu fiable pour le futur historien. Pour prendre un exemple qui a l'inconvénient d'être personnel mais l'avantage que je puis en juger directement, Mendras affirme: "Stoetzel envoie Boudon chez Lazarsfeld à Columbia”. Personne ne m'a envoyé à Columbia et surtout pas Stoetzel que je n'avais pas encore rencontré et qui ignorait alors jusqu'à mon existence» (Boudon, 1996). 
correspondance disponible entre ces trois sociologues. L'article met l'accent sur l'importance de ce que ce matériau original met au jour quant aux processus d’apprentissage, de réputation et de mobilité académique. Il souligne également la nature de l'ethos scientifique partagé par les trois sociologues.

\section{LE FONDS RAYMOND BOUDON}

Pensé dès l'origine comme une contribution à l'élaboration d'un futur centre d’archives de la recherche en sciences sociales (Sène, 20I4), le fonds Boudon a été déposé en 2013 aux Archives nationales. La série d’entretiens réalisée avec Brigitte Mazon pour la constitution de ce fonds (Boudon, 20I3) a été l'occasion pour Boudon de retracer les principaux moments de sa carrière, mais également d'évoquer la nature même de ces archives. À la question «Que pensez-vous que contiennent vos archives? », il répond:

Sans doute surtout du quotidien, c'est-à-dire tout ce qui fait la vie d'un enseignantchercheur: des rapports de thèses, des évaluations de candidatures, des lettres de recommandation, des documents d'ordre administratif, des demandes de crédit, etc. Des choses peut-être plus originales aussi [...]. Mais l'essentiel des archives, c'est sans doute de la routine. Je ne crois pas que ce soit inintéressant pour autant, car, si un jour un historien venait à écrire l'histoire des sciences sociales de la seconde moitié du $\mathrm{xx}^{\mathrm{e}}$ siècle, cela pourrait lui être utile (ibid., p. 608).

Le catalogue des archives suggère que cette « routine » de la vie académique est, comme on peut s'y attendre, multidimensionnelle. Le fonds Boudon s'organise autour de six grandes catégories de documents. La première concerne les activités institutionnelles au sein du CNRS et autres organismes de recherche nationaux et internationaux ainsi qu'à l'Académie des sciences morales et politiques. La deuxième concerne les activités d'enseignement et d'encadrement dans des contextes institutionnels variés: l'université de Paris V Descartes, l'université Paris IV Sorbonne, l'université de Genève, l'université de Harvard, etc. Sont réunies ici notamment les différentes contributions de Boudon aux rapports de soutenance de thèse auxquelles il a eu l'oc- 
casion de participer ${ }^{5}$. La troisième catégorie concerne les missions, colloques et interviews. On y trouve notamment les traces d'une première demande de visa permanent pour les USA en 1969 ainsi que les textes d'interventions produits lors de colloques et conférences en France comme à l'étranger. La quatrième catégorie regroupe les différentes publications et tirés à part sur la période 1962-20II. Le fonds regroupe aussi l'ensemble des ouvrages écrits ou co-écrits par Boudon, et leurs diverses traductions, les entretiens et compte rendus parus dans la presse à l'occasion de la sortie de ces ouvrages. La cinquième catégorie est centrée sur l'activité éditoriale de Raymond Boudon : contrat d'édition, dossier de presse, préparation des manuscrits, relations avec les éditeurs, et particulièrement bien entendu avec les Presses universitaires de France pour le suivi quotidien de la célèbre collection bleue Sociologies (Langlois, 2008). Enfin, la sixième catégorie, celle qui nous intéressera ici plus longuement, concerne la correspondance générale, reçue ou expédiée.

Boudon fait-il partie de la communauté des sociologues épistoliers? Sa réponse était simple et directe : «il n’y a pas dans mes archives de correspondances à proprement parler. [...] J'ai eu surtout des conversations avec plusieurs personnes [qui] ont eu pour effet de me conduire à approfondir certaines idées et certains textes et à en corriger d'autres» (Boudon, 20I3). Faut-il pour autant considérer que cette absence d'intérêt pour la relation épistolaire - qui

5 La liste de ces contributions aux rapports de soutenance de thèse telle qu'elle apparaît dans l'inventaire permet de dessiner les traits d'un collectif, avec par ordre alphabétique: Albertina Oliverio, Abdelmajid Arbouche, Enric Becescu, Daniel Benamouzig, Henri Bergeron, Emmanuelle Betton, Martin Blais, Raymond Bourdoncle, Alban Bouvier, Alain Boyer, Gerald Bronner, Nathalie Bulle, Mme Chchenkova, M. Choi, François Cusin, M.Damoiselet, Pierre Demeulenaere, Jacqueline Deguise-Le Roy, Lyliane Gurcel Deroche, M. Dies, Annette Disselkamp, Michel Dubois, M. Dumaitre, Patrice Duran, Joseph Facal, Mme Fericelli, Renaud Filieule, M. Friedberg, Frédéric Gerard, Claude Giraud, Francine Gratton-Jacob, Benoît Grison, Alexandre Guillard, Dominique Guillo, Boris Guroy, Henri Bergeron, M.Hyun, Agata Jackiewicz, Loic Jarnet, Marc Lambret, Lefebvre, Philippe Lefebvre, Marc Le Menestre, Marc Leroy, Eric Letonturier, Carlo Lottieri, Raul Magni-Berton, Gianluca Manzo, Jean-Christophe Merle, Michael Ballé, Bruno Milly, Lucien Samir Oulahbib, Maxime Parodi, Dunia Pepe, Emmanuel Picavet, Jocelyn Raude, Renaud Emmanuel, Sandra Roquet, Michel Routon, Bertrand Saint-Sernin, Kei Tsujihara-Sakata, Chang Hack Shim, Simoniatova, Emmanuel Plot, Gérard Spiteri, Anne Staszak, Philippe Steiner, Taleb-Khyar, Teller, Dominique Terré-Fornacciari, Laurent Tessier, Patricia Vanier, Fiorella Vinci. 
se manifeste tout de même paradoxalement par un volume non négligeable de I cartons d'archives de correspondance ${ }^{6}$ - ne peut rien nous apprendre sur la trajectoire sociologique de Boudon et plus largement sur la vie académique française et internationale? On peut en douter, notamment lorsqu'invité à évoquer ses «amitiés intellectuelles» Boudon en vient à rappeler sa relation avec le duo Lazarsfeld-Merton : «vous trouverez peut-être dans les archives quelques lettres de Lazarsfeld, peut-être quelques lettres de Merton. Je ne sais pas si vous y trouverez une lettre où Merton écrit à Lazarsfeld qu'il avait l'impression quand il me lisait (lui, Merton) que j'écoutais leurs conversations à Columbia. Lazarsfeld a pensé que cela me ferait plaisir de l’avoir et me l’a envoyée » (ibid.).

Avant d'en venir à la description plus détaillée de cette correspondance entre les trois hommes, il est utile de donner une représentation d'ensemble, même sommaire, de cette correspondance générale de Boudon et du travail d'exploitation qui a été entrepris à partir de l'automne 2014.

L'exploitation de ces onze cartons d’archives de correspondance a été réalisée en deux temps. Dans un premier temps dans les locaux même du service des archives de l'EHESS, puis une fois le fonds Boudon basculé aux Archives nationales, sur le site de Pierrefitte-sur-Seine des Archives. L'objectif initial, formulé en accord avec la direction du GEMASS, le laboratoire créé par Boudon au début des années $1970^{7}$, était de dépouiller et inventorier l'intégralité de la correspondance pour pouvoir par la suite réaliser un certain nombre de traitements. À ce jour un corpus épistolaire d'environ 2150 items (courriers reçus, expédiés, en copie, etc.) a d'ores et déjà été constitué pour la période I96I-200I. Près de $40 \%$ des items présents dans le corpus ont été expédiés par Boudon, les $60 \%$ restant étant des items dont il était le destinataire principal ou secondaire (notamment pour les items en copie).

6 Un volume qui demeure certes limité au regard des correspondances disponibles de certains sociologues-épistoliers tels que Merton (Dubois, 2014).

7 Voir à ce sujet les pages dédiées aux archives Boudon sur le site du GEMASS: <https://www. gemass.fr/les-archives-du-gemass-et-de>. 
Figure I. distribution du corpus, nb de correspondants par année, nb d'items par année (I96I-200I)

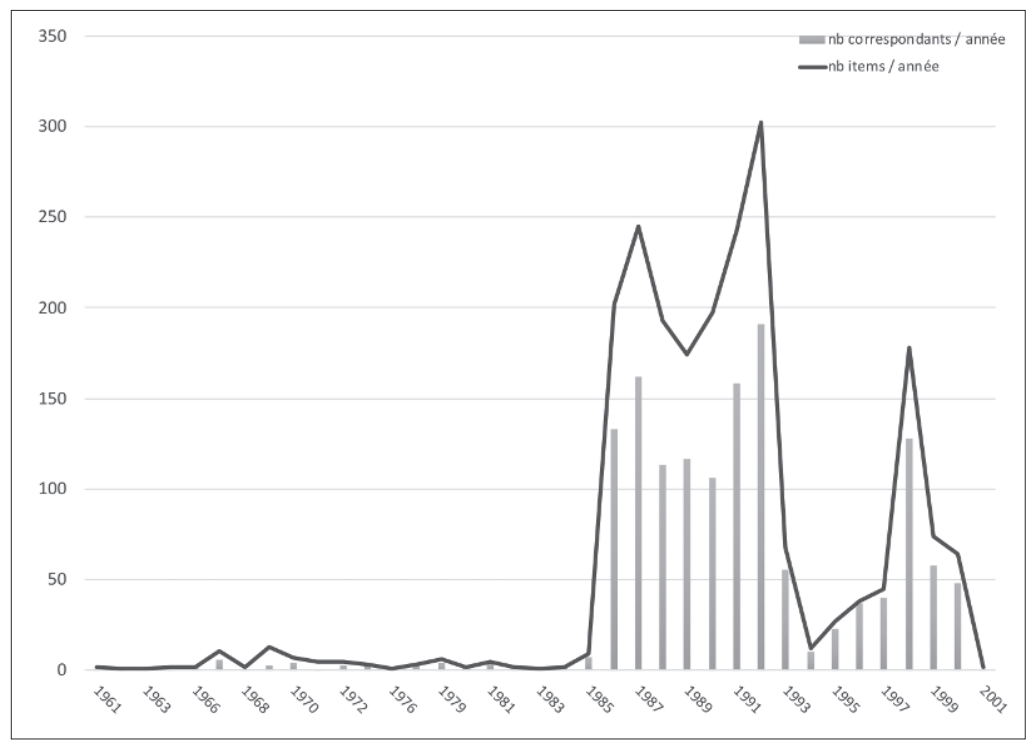

La figure I donne une représentation d'ensemble du corpus (par nombre d'items et nombre de correspondants) et de sa distribution dans le temps. L’essentiel de la correspondance présente dans notre corpus général porte sur la période des années 1980-2000. L’écart observé sur certaines périodes entre le nombre de correspondants et le nombre d'items s'explique aisément par l'existence de relations épistolaires régulières avec un nombre limité de correspondants. L'utilisation de l'approche relationnelle des correspondances académiques d'ores et déjà exploitée à différentes échelles - méso (Dubois, 20I4) et micro (Dubois, 20I4a) - permet de construire une représentation d’ensemble du corpus (voir Figure 2) mais également, de façon plus informative, de préciser la nature du collège épistolaire de Boudon (voir Tableau I). 
Figure 2. visualisation du corpus de correspondance générale de Raymond Boudon comme réseau personnel, Algorithme Fruchterman Reingold, $\mathrm{n}=902$

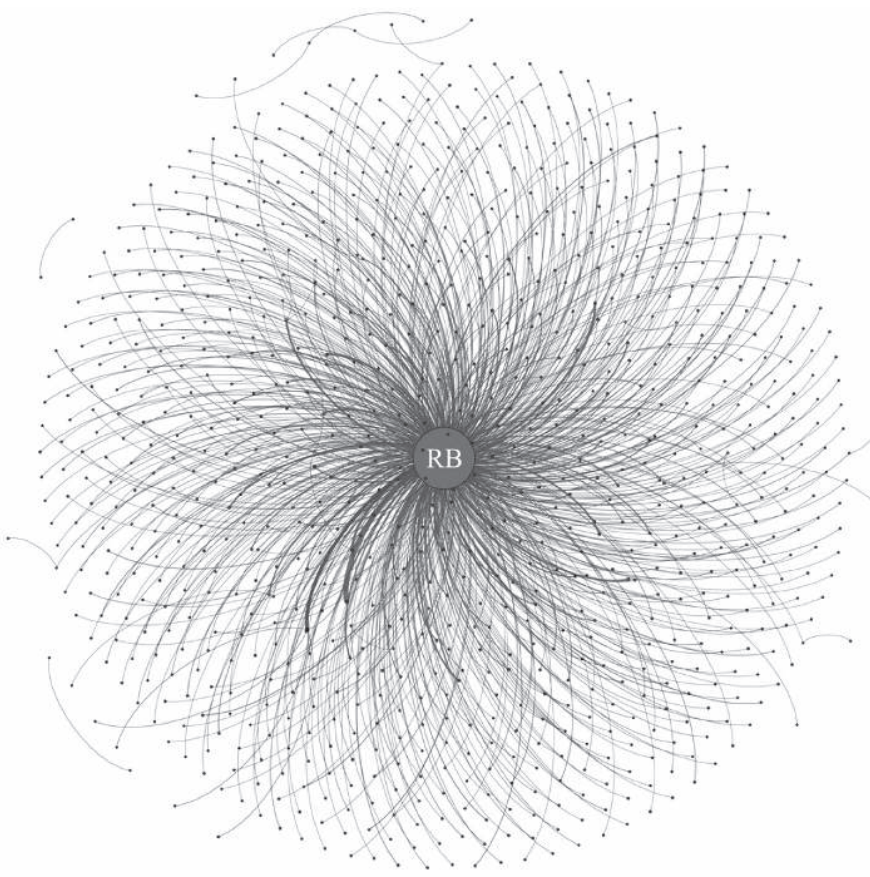

Tableau i. Le collège épistolaire de Boudon, $\mathrm{n}=40$, par ordre de fréquence décroissante (rangs I à 40)

\begin{tabular}{|l|l|l|l|l|l|l|c|}
\hline \multicolumn{1}{|c|}{ ID } & RANG & \multicolumn{1}{|c|}{ ID } & RANG & \multicolumn{1}{|c|}{ ID } & RANG & ID & RANG \\
\hline busino-g.. & 1 & coenen-huther-j. & II & casanova-j.c. & 21 & drouard-a. & 31 \\
\hline lazarsfeld-p. & 2 & peyrefitte-a. & 12 & pellicani-l. & 22 & forsé-m. & 32 \\
\hline merton-r.k. & 3 & helle-h. & 13 & poussou-j.. & 23 & lautman-j. & 33 \\
\hline eisenstadt-s.. & 4 & saint-sernin-b. & 14 & barker-p. & 24 & lazar-j. & 34 \\
\hline bouvier-a.. & 5 & bronner-g. & 15 & berthelot-j.m. & 25 & lindenberg-s. & 35 \\
\hline prigent-m. & 6 & morin-j.m. & 16 & birnbaum-p. & 26 & marot-g. & 36 \\
\hline bunge-m. & 7 & paqueteau-b. & 17 & massot-a. & 27 & montbrial-t. & 37 \\
\hline bell-d. & 8 & rezsohazy-r. & 18 & rotariu-t. & 28 & picavet-e. & 38 \\
\hline assogba-y. & 9 & terré-d. & 19 & sakata-k. & 29 & pithod-a. & 39 \\
\hline leroy-m. & 10 & boyer-a. & 20 & delara-p. & 30 & renaut-a. & 40 \\
\hline
\end{tabular}


Sans entrer dans le détail de différents noms présents dans ce tableau I - certains bien connus d'autres moins connus -, il est utile pour notre propos de faire deux observations: I) une partie importante de ces noms est liée au milieu académique national dans ses dimensions universitaires ou éditoriales ; 2) pour ce qui concerne la part des collègues étrangers présents dans ce collège épistolaire, Lazarsfeld et Merton occupent des places de choix, respectivement au deuxième et troisième rangs. La correspondance d’amitiés intellectuelles entre Boudon, Lazarsfeld et Merton - constitue donc bien une composante importante de la correspondance générale de Boudon, et à c'est à ce sous-ensemble et à la relation entre ces trois sociologues qu'il faut désormais s'intéresser.

\section{ENTRE PARIS ET NEW YORK: UNE CORRESPONDANCE D'AMITIÉS INTELLECTUELLES}

La correspondance de Raymond Boudon avec les deux «complices » de l'université Columbia à New York (Boudon, 2010) s'étend sur une période assez longue puisqu'elle débute dans les années 1960 pour s’achever au début des années 2000.

Le fonds d'archives contient 34 lettres adressées par Lazarsfeld à Boudon du I ${ }^{\text {er }}$ décembre 1965 au I I août 1976 et I6 lettres envoyées par Merton du II juin 1970 au 7 novembre $200{ }^{8}$. Ces correspondances ont été l'une comme l'autre interrompues par le décès, en août 1976 de Lazarsfeld, et en février 2003 de Merton. Par leur durée comme leur contenu, elles manifestent une affinité élective et cognitive profonde. Une affinité qui s'est maintenue tout au long du parcours intellectuel de Boudon, de la thèse sur l'analyse mathématique des faits sociaux à la formation du paradigme de l'individualisme méthodologique et à son extension progressive vers une théorie de la rationalité élargie intégrant la rationalité axiologique.

8 À ces lettres, il faut ajouter celles envoyées en copie à Boudon par Lazarsfeld et/ou Merton. Il est à noter que l'essentiel de la correspondance archivée a comme expéditeur Lazarsfeld et Merton. II n'existe pour cette correspondance que rarement une copie des courriers expédiés par Boudon lui-même. 
Ces correspondances rendent également fréquemment compte de l'évolution du milieu professionnel environnant en France comme aux États-Unis. Et c'est à ce double titre - formation d'un paradigme sociologique en France, transformation nationale et internationale des cadres disciplinaires - qu'elles méritent d'être examinées aujourd'hui, tant par les historiens des sciences sociales que par les sociologues eux-mêmes.

Cet échange épistolaire débute au milieu des années 1960, à un moment où les sciences sociales connaissent en France un double mouvement d'institutionnalisation et de professionnalisation. La période est également marquée par un accroissement des grands investissements financiers et organisationnels des fondations américaines - Carnegie, Rockfeller, Ford, etc. - et d’autres institutions internationales (comme l'UNESCO) afin de faciliter et d’accélérer l'intégration de l'enseignement et de la recherche, et enfin une intensification des échanges entre les deux rives de l'Atlantique (Drouard, 1982; Miéville et Busino, 1996). Revenant sur la situation de la sociologie française durant cette période, Boudon résumera ainsi la représentation qu’en avait le jeune normalien de l'époque : «trois hommes faisaient la pluie et le beau temps dans la sociologie française: Aron, Stoetzel et Gurvitch» (Boudon, 20I3, p. 345). Tous trois sont alors les grands patrons de la sociologie à la Sorbonne où une licence de sociologie a été créée en 1958 et une référence obligée pour tous ceux qui souhaitent étudier dans ce domaine. Georges Gurvitch, unique professeur de sociologie à la Sorbonne dès 1948 avant l'arrivée de Raymond Aron, s'impose à l'époque comme un personnage clef de la sociologie française (Marcel, 200I). Ce dont témoignera Boudon, devenu lui-même plus tard une grande figure internationale de la sociologie: «Il avait la seule chaire de sociologie à la Sorbonne, dirigeait la seule collection de livres de sociologie aux Presses universitaires de France, «la Bibliothèque de sociologie contemporaine». Il présidait à la seule revue de sociologie florissante à cette période, les Cahiers internationaux de sociologie. Il régnait en despote sur la sociologie française». (Boudon, 2013, p. 344). Aron, de son côté, entré à la Sorbonne en 1955, vient de créer son Centre européen de sociologie (1960), ainsi que les Archives européennes de sociologie (1960) devenues aujourd'hui le European Journal of Sociology (Heilbron, 2015, p. I72). Quant à 
Jean Stoetzel, dont Boudon confiera qu'il était son «véritable maître »en France (Boudon, 2003, p. 38)9 , il était entré à la Sorbonne en 1955 comme Aron sur une chaire de psychologie sociale, après avoir créé en 1938 le premier institut de sondage en France largement inspiré des Survey Research de Lazarsfeld, l'IFOP. Il était également à l'origine de la création, en 1960, de la Revue française de sociologie dont la vocation était de publier les résultats de recherches à caractère empirique.

Boudon n’a jamais caché sa réticence à l'égard de la sociologie d’un Gurvitch, ni même son indifférence à celle pratiquée par Aron: «Je voyais bien que la sociologie de Georges Gurvitch était respectable par l'énergie qu'elle révélait et qu'elle contenait, mais qu'elle n'avait pas beaucoup d'avenir. Raymond Aron me paraissait être surtout [...] un grand intellectuel. Il mavait lui-même déclaré que, en matière de sciences sociales, il se voyait comme un vulgarisateur ». (ibid., p. 38). C'est donc tout naturellement vers Lazarsfeld et son ami et «pendant» théorique français, Stoetzel, que se dirigèrent les espoirs du jeune normalien féru de mathématiques et épris de rigueur scientifique ${ }^{10}$.

L'école de Columbia, organisée autour de Lazarsfeld et de Merton, était alors à son apogée (ibid., p. 37 ; Pollak, 1979). Tandis que la sociologie fonctionnaliste de Parsons connaissait un déclin à partir des années 1950 et que la célèbre école de Chicago commençait à s'essouffler, la sociologie empirique et quantitativiste de Lazarsfeld fondée sur l'emploi intensif des mathématiques et des statistiques mobilisées au service d'une «analyse empirique de l'action » gagnait en visibilité scientifique et en importance institutionnelle. Au début des années 1960, le Bureau of Applied Research, créé par Lazarsfeld, et l'université de Columbia étaient le centre le plus prestigieux des États-Unis. Revenant sur sa carrière et son parcours intellectuel, Boudon observait sous forme de boutade: «La sociologie scientifique existe: je l'ai rencontrée» (Boudon, 20ı, p.4). On peut dire que dans ces années-là, il l’avait rencontrée en la personne de Lazarsfeld.

9 C'est au fauteuil laissé vacant par Stoetzel que Boudon sera élu le 29 mai 1990 à l'Académie des sciences morales et politiques. Voir «Notice sur la vie et les travaux de Jean Stoetzel», séance du 18 février 1992.

10 Sur le rapprochement théorique et méthodologique entre Lazarsfeld et Stoetzel, voir Blondiaux, 1990. 
Ayant obtenu par l'intermédiaire d'Aron une bourse de la Fondation Ford, il décida dès lors, comme Stoetzel l'avait fait d'ailleurs avant lui, de se rendre à Columbia où il passa l’année I96I-I962:

Personne ne m’a envoyé à Columbia, mais j’avais décidé de faire un stage chez Lazarsfeld à l'issue de mon service militaire, parce que j'avais découvert un peu par hasard sur les rayons de la bibliothèque de la rue d'Ulm The Language of Social Research. À tort ou à raison, ce livre m'était apparu comme offrant une alternative à la sociologie gurvitchienne alors régnante en France, que j'avais toujours soupçonnée de dissimuler beaucoup de banalités dans des maquis de typologies et de définitions tatillonnes, et à sa concurrente structuraliste, qui me paraissait sujette à caution, tant par son ambition totalisante que par son platonisme (Boudon, 1996, p. 77).

Le dialogue, amorcé dès le début des années 1960, entre Boudon et les patrons du département de sociologie de Columbia demeurera intense et vivant pendant de nombreuses années. Mais un tel dialogue ne se limite pas à la seule correspondance, qui n'en n'est qu'une partie immergée. La circulation scientifique transnationale s'effectue aussi à travers des flux d'hommes, de méthodes et d'idées (Chaubet, 20I4) et le dialogue entamé entre Boudon et les penseurs de Columbia n'échappe pas à la règle. On sait ainsi que Boudon, dont Aron disait qu'il représentait «une sorte d'îlot de sociologie américaine sur le territoire français» (Boudon, 20ıо, p. I3), a effectué de nombreux séjours aux États-Unis : «J’ai vécu à plusieurs reprises aux ÉtatsUnis, une première fois comme étudiant à Columbia pendant un an, ensuite en 1972 comme fellow au centre de Palo Alto en Californie. J’ai été invité dans plusieurs universités; entre autres à Harvard en 1973, à Chicago en 1986, à l'université d'Indiana, à l'université de New York» (Boudon, 20ı3, p.607). On sait aussi que Lazarsfeld, né à Vienne, et très soucieux d'exporter en Europe sa conception de la sociologie, s'est rendu de très nombreuses fois en France (Gemelli, 1998 ; Lécuyer, 2002). Amorcée dès 1948 dans le cadre d'un séminaire du Centre d'études scientifiques de la politique intérieure de la Fondation nationale des sciences politiques sur l'invitation de son président, André Siegfried, la fréquentation de la France par Lazarsfeld atteint un pic au milieu des années 1960, dans le cadre de l’Unesco tout d’abord en 1960, puis 
pendant deux longues périodes d'enseignement à la Sorbonne à l'initiative de Jean Stoetzel, en 1962-1963 et 1967-1968, pour trouver son point culminant quand la Sorbonne lui attribue le titre de professeur émérite.

La circulation transatlantique des idées scientifiques s'effectue aussi à travers la production d'ouvrages, leur traduction et leur indispensable acclimatation aux contextes intellectuels respectifs. Durant la période 1965-1976, soit celle qui couvre la période de la correspondance avec Lazarsfeld disponible dans les archives, Boudon a déjà publié, outre ses deux thèses, L'Analyse mathématique des faits sociaux (1967) et À quoi sert la notion de structure? (1968), Les Méthodes en sociologie (1969), La Crise de la sociologie (197I) et enfin l'ouvrage grâce auquel il acquiert une véritable notoriété scientifique internationale: L'Inégalité des chances (1973). Lazarsfeld a supervisé de près l'élaboration des deux thèses de Boudon comme la qualité de la traduction de ses différents travaux afin qu'ils soient reçus dans les meilleures conditions possibles par la communauté scientifique américaine. Réciproquement, Boudon contribuera à diffuser la pensée de Lazarsfeld en France à travers la co-direction de plusieurs de ses livres: Le Vocabulaire des sciences sociales. Méthodes de sociologie, vol. I (1965), L’Analyse empirique de la causalité. Méthodes de sociologie, vol. II (1966), et, avec François Chazel, L’Analyse des processus sociaux. Méthodes de Sociologie, vol. III (1970), ainsi qu'en publiant des morceaux choisis de Lazarsfeld, une première fois en français en 1970, et une seconde fois en anglais, en 1993, après la mort de ce dernier (Lazarsfeld, 1970; Lazarsfeld, 1993).

Quant au lien avec Merton, on sait par Boudon (2010, p. I3) qu'ils continuèrent d'échanger leurs publications et de parler de leurs travaux jusqu'à la fin de l'année 2002, peu avant le décès de Merton. Comme l'a rappelé Lécuyer (2002), l'arrivée à Columbia de Merton - la même année que Lazarsfeld (en 194I) - a été la conséquence de l'impossibilité de départager deux figures du département de sociologie de l'époque, Robert MacIver, sociologue théoricien et Robert Lynd, méthodologue. Le premier cherchant à faire recruter Merton, le second Lazarsfeld. Plutôt que de trancher, les responsables de Columbia choisirent de dédoubler le poste en recrutant simultanément Lazarsfeld et Merton. Cette complémentarité entre ces deux hommes et, à travers eux, entre les deux dimensions de l'analyse sociologique - la méthodologie d’une part, la théorie 
d’autre part - a profondément marqué Boudon: «Nous avions [...] l'impression que le duo Lazarsfeld-Merton proposait un exemple de collaboration remarquable » (Boudon, 2003, p. 390) ; ou encore (dans l'un de ses derniers ouvrages): «La symbiose entre l'inspiration méthodologique de l'un et la tendance théorique de l'autre m’impressionna» (Boudon, 2oı, p. 9).

Contre les approches globalisantes de la société, Boudon voyait en Merton celui à partir duquel il devenait possible de repenser les échelles de l'analyse sociologique: «Merton nous avait tous convaincus que la notion de Middle range theory (théorie à moyenne portée) soulevait une question essentielle en ce qu'elle oppose les théories expliquant des phénomènes bien définis aux "théories" qui prétendent traiter de la société en général » (ibid). Et de fait, une part importante de la théorie mertonienne de l'action et de ses conséquences non intentionnelles sera par la suite au cœur de ses ouvrages, tout particulièrement Effets pervers et ordre social (1977) ou La Logique du social (1979). Merton était parfaitement conscient de l'importance de la contribution théorique originale de Boudon - «Your book on effets pervers strikes me as being of the first importance» (lettre de Merton à Boudon datée du I5 mai 1979) - même s’il lui arrivait parfois de s'inquiéter, non sans humour, des conséquences éditoriales que la circulation internationale de ses publications pouvait avoir à terme pour ses propres projets ${ }^{11}$.

II Comme dans ce courrier du 21 juin 1982 dans lequel Merton fait part de son étonnement suite à la traduction anglaise de l'ouvrage Effets pervers et ordre social: «Dear Raymond, It was kind of you to have a copy of the english translation of your Effets pervers sent to me [...]. I was a bit startled and, for a time, put off - just as you must have been when you discovered that the English publishers adopted the title of Unintended consequences of social action. Let's hope that this title is not also adopted by an American publisher. It doesn't quite preempt the title of the volume I have been editing, so perhaps no damage will have been done. I know how publishers sometimes make decision without bothering to notify authors about them and so we'll both have to live with it as a fait accompli. In a way, this is also poetic justice. After all, the volume on Unanticipated Consequences should have been in print at least two years ago, were it not for a variety of events that intervened »). 


\section{3. «IN THE MERTON-LAZARSFELD SPIRIT» : PROFESSIONNALISATION ET ETHOS SCIENTIFIQUE}

À un niveau personnel, ce qui frappe tout d'abord à la lecture de cette correspondance d'amitiés intellectuelles, c'est le ton chaleureux des échanges entre les trois hommes: «Dear Raymond», «Dear Paul», «Dear Bob», etc. On perçoit aisément à travers les lettres échangées, le plaisir de se revoir, la déception de s'être manqué, la trace d'une amitié qui se construit, le témoignage d'un dialogue continu dont la correspondance ne reflète qu'une infime partie. Sillonnant l'Europe et effectuant de nombreux aller et retour entre la France et les États-Unis, on voit ainsi Lazarsfeld rédiger dans l'avion qui le ramène à Columbia une lettre destinée à poursuivre sans attendre l'échange qu'il venait d'avoir avec Boudon à Paris. «Dear Raymond, On my flight back I read your new chapter very carefully and I want to comment on one point in some detail. » (Lettre du $\mathrm{I}^{\text {er }}$ novembre 1965).

Les trois sociologues se tenaient en très haute estime. C'est aussi ce que reflète cette correspondance. Boudon a dit toute l'admiration qu'il éprouvait pour Lazarsfeld, qu'il considérait «comme son maître» (Boudon, 20I3, p. 346), comme il a évoqué à de multiples reprises le respect que lui inspirait Merton. On lit dans ces courriers adressés à Boudon que ce sentiment était réciproque. Merton, par exemple, tient à exprimer à Boudon, dans une lettre datée du 27 mars 199I, combien il partage le sentiment de Mario Bunge selon lequel il fallait voir en lui, avec James S. Coleman, l'un des «very best brains in all of social science». Ce respect et cette admiration réciproques se donnent à voir également dans les réactions qui accompagnent la réception des premiers travaux de Boudon. Ainsi, à propos de sa thèse, Lazarsfeld peut-il écrire : «It is a compliment to your thesis that I keep on thinking about it» (Lettre du I4 juin 1966). Écrivant quelques années plus tard à l'éditeur américain de L’Analyse mathématique des faits sociaux, il en souligne l'importance à ses yeux : «I was always sure that Boudon's book is very important. It would have a very salutary effect in this country. There are so many mathematical sociologists that break up in little cliques favoring one method or another. Boudon shows that most of these trends are just special cases of the more general idea which he presents competently» (Lettre du $\mathrm{I}^{\mathrm{er}}$ mars 197I). 
Par-delà cette dimension personnelle d'affinité et de respect mutuel, l'étude de cette correspondance met en lumière différentes dimensions étroitement liées aux années d'apprentissage et de socialisation professionnelle de Boudon. Ce dernier a dit qu'il considérait Lazarsfeld comme son «mentor», et c'est bien comme tel qu'il apparaît dans ces courriers échangés. Dès son retour de Columbia, Boudon décide de faire sa thèse principale sur l'apport des mathématiques aux sciences sociales sous la direction de Stoetzel et, sur la suggestion de Lazarsfeld, de consacrer sa thèse secondaire à la notion de structure avec comme directeur Aron.

De nombreuses lettres témoignent du fait que Lazarsfeld prenait son rôle de mentor très au sérieux. Évoquant ses années d’apprentissage, Boudon a souligné la rigueur de Lazarsfeld : «Il était un redoutable directeur de thèse. Il a fait refaire trois fois sa thèse à un ami américain pour finalement lui refuser l'accès à soutenance» (Boudon, 20I3). L'étude de la correspondance entre les deux hommes montre que Lazarsfeld lisait très méticuleusement la thèse de Boudon, n'hésitant pas à lui faire préciser certains points, à être plus rigoureux sur son concept de «structure » de manière à bien le définir par rapport à ce que «our enemies » entendent eux-mêmes par ce terme (Lettre du I4 juin 1966). Certaines de ces propositions pouvaient être plus exploratoires, comme par exemple lorsqu'il invite Boudon à tenter d'imposer en France la notion de «sondage sociologique » comme équivalent de l'expression «empirical social research»:

After all, the word «sondage» had a more general meaning before it was occupied by the public opinion people. It has a connotation of general inquiry by soundings that means indicators and this is, after all, what we do. While you cannot help that sondage now means for many public opinion research by sampling, you have a good chance if you turn around and now call sondage sociologique all the other types of empirical soundings on contemporary social topics, irrespective of specific method used (Lettre du $\mathrm{I}^{\text {er }}$ novembre 1965).

Mais c'est surtout à l'occasion de la préparation de la traduction, restée inaboutie $^{12}$ de L’Analyse mathématique des faits sociaux (d’après la $2^{\mathrm{e}}$ édition de 1970) que l'on peut mesurer l'investissement de Lazarsfeld. Constatant que la traduction initiale était «catastrophique», il n’hésite pas à retraduire lui-même une 
partie du texte, ce qui ne semble d'ailleurs pas lui avoir coûté : «I enjoy working on the translation because it forces me to think through a lot of matters to which I have not given enough attention before. Thus, for instance, I try to develop a direct derivation of what you caIl complex structures without interaction; I mean a derivative that comes directly from your decomposition of probabilities. I think I am on the right way but in any case, your book should not be burdened with this» (Lettre du 9 mars 197I).

Soucieux de l'accueil du livre de Boudon auprès d'un public familiarisé avec l'analyse empirique et statistique, c'est surtout sur le fond de l'argumentation développée que Lazarsfeld se montre d'une très grande exigence. Les lettres de cette période sont souvent accompagnées de longs memorandums écrits à la main par Lazarsfeld dans lesquels il demande à Boudon de reprendre en profondeur telle ou telle argumentation, tel ou tel passage : «Please reread your chapter 3 because it is for the most important for the whole strategy of the publication » (Lettre du 22 mars 197I). Extrêmement pointilleux sur l'exigence de rigueur scientifique, il ne lui «laisse rien passer», tout en prenant soin de ne pas trop le froisser : «I was glad to learn that my comments were useful [...] You will see that nowhere do I disagree with your findings. I am essentially concerned with matters of presentation. But for an American edition this is important because you have here a public which is much better prepared for your book than the French» (Lettre du 27 octobre 1969).

Cette sévérité du mentor Lazarsfeld n'est cependant que l'envers de son soutien indéfectible à la diffusion des travaux de Boudon aux États-Unis. Et à l'occasion de la parution américaine de L'Inégalité des chances, Lazarsfeld aura l'occasion de dire tout le bien qu'il pensait de la réponse faite par Boudon à Robert M. Hauser (Hauser, 1976; Boudon, 1976). Une lettre que Boudon aimait à citer: « Thank you very much for sending me the debate between you and Hauser. I was

choix initial du traducteur par Lazarsfeld lui-même: «I was responsible for Little, Brown having commissioned Dr Kenneth Land for the translation. His wife is French-Belgian and he is a well-trained mathematical social scientist. I promised Boudon to go over the final English translation from a didactical point of view [...]. I got a copy a little while ago. It is mere coincidence that I became aware of the language problem [...]. The translation is still abominably bad [...]. I would advise you and urge Boudon not to proceed with the publication of the book without a thorough revision » (Lettre du I mars 197| de Lazarsfeld à Alfred L. Brown). 
impressed by your reply and angry with Hauser. He is a very typical example of statistical zealot although he is undoubtedly competent. » (Lettre du I5 août 1975).

Cette correspondance montre aussi de quelle manière Lazarsfeld, dans les années 1970, puis Merton, dans les années 1980, ont contribué chacun à leur manière à établir et renforcer la réputation comme la visibilité de Boudon dans la vie académique aux États-Unis. En 1976, Lazarsfeld participe, dans le cadre de l’American Sociological Association, au comité chargé de décerner le «Stouffer award», un prix récompensant les meilleures contributions méthodologiques en sociologie. Lazarsfeld a obtenu cette récompense en 1973, James S. Coleman en 1975 et c'est donc très logiquement que Lazarsfeld prend rapidement contact avec Boudon pour lui demander de transmettre des éléments de candidature :

There is a reasonable chance that you will be one of the awardees. It would be of great help if you were to send me a list of your publications - including translations - and your guest appearances like your stay at Harvard. You have to understand that the final outcome of such competition often depends upon quite unforeseeable circumstances. So what I am writing you here is a reasonable guess, but by no means a firm forecast. Still it would be worthwhile if you were to send me the material I just mentioned (Lettre du zo juin 1976).

Quelques années plus tard, Merton jouera un rôle similaire pour faire venir Boudon à l'université Columbia. Ce dernier est invité, en février 1983, à prononcer une conférence prestigieuse : la $5^{\mathrm{e}}$ «Paul Lazarsfeld Lecture». Merton est alors en charge de l'organisation de l'événement: «Dear Raymond, Interest is mounting in your giving the Fifth Paul Lazarsfeld Lecture. So much so, that we are eager to give it the greatest possible advance publicity - to colleagues, Paul's many former students and longtime friends, the entire Columbia community, and social scientists from Boston and Washington, Some word-of-mouth has gotten round and we have had inquiries about the exact date and other such details in recent weeks. » (Lettre du 27 septembre 1982). Une semaine à peine après la conférence, Merton fait part de son enthousiasme, comme celui de ses proches, à Boudon: «Even in short retrospect, it seems that the blizzard of '83 did nothing to dim the occasion of your lecture. Quite the contrary; even now, members of that hardy crew who made it through the deepening snow to Low Rotounda are talking of their experience with evident relish. We can count, I think, on this event being told and retold for many years to come. I trust that you had a sense of the warm and interested response to your lecture ». Et Merton d'ajouter 
à l'intention de Boudon un message en forme d'invitation à venir le rejoindre définitivement à Columbia : «All of us here enjoyed your stay beyond easy description. $A$ pity that you can't manage a truly extended time with us - say, a semester or an academic year (to say nothing of your joining with us for good) » (Lettre du I8 février 1983).

Par-delà cette logique de réputation et de mobilité académique, c'est surtout de façon plus générale un même ethos scientifique qui se donne à voir à travers cet échange épistolaire entre les trois hommes. Ce que Boudon avait trouvé chez Lazarsfeld et Merton, c'était avant tout l'idée qu'il pouvait exister une «analyse scientifique de l'action» (Boudon, 1998, p. 37I) et une «sociologie centrée sur l'individu» (Boudon, 1970, p.4I) et organisée autour de ses décisions. Affirmant qu'il s'était toujours reconnu dans «une conception scientifique de la sociologie», Boudon confia n’avoir jamais eu de difficulté à comprendre la moindre phrase de Lazarsfeld tant il parvenait à circuler dans son univers mental avec aisance (Boudon, 1996, p. 76).

C'est ce que confirme une lettre de Merton adressée à Lazarsfeld à propos de l'introduction de Boudon à sa Philosophie des sciences sociales, et que ce dernier lui transmit non sans fierté :

Dear Paul, When you get home from your Parisian triumph - not mere ovation - I want you to know how much I liked Boudon's introduction to your book. It is splendid. It has caught your «obsessions» right down to their roots [...] it is almost as though has had been there through the years, listening to some of our endless conversations and now hearing your own insistence on what really matters. Boudon's introduction belongs in the same class with your introduction to sam's book and that is saying a great deal. Not least, his French is as crisp and clear as your written English (Lettre du II juin 1970).

Dans une lettre à Merton du 24 février 1993, Boudon redit toute son affinité pour ce qu'il décrit comme étant le «Merton-Lazarsfeld spirit». Cet esprit, c'est à ses yeux celui des concessions minimales aux idéologies du moment ainsi quaux valeurs hic et nunc. Cet esprit, c'est également, et surtout, le choix de faire de la création de connaissance la fonction première de la discipline. Et c'est sans nul doute ce choix, central pour Lazarfeld comme pour Merton, qui traverse les pages consacrées à Columbia par Boudon dans son autobiographie intellectuelle intitulée précisément La Sociologie comme science: «Les études produites autour du groupe des sociologues de Columbia, rappelle-t-il, m’ont séduit parce qu’elles étaient créa- 
trices de savoir [...] elles se concentraient sur des sujets concrets et circonscrits. [elles] réussissaient à frôler l'universel à travers le singulier » (Boudon, 20 Io, p. I I).

L’attention accordée par Boudon et Merton à l'«universalisme» comme idéal régulateur de l'activité scientifique explique en grande partie le fait que, dès le début des années $1990^{13}$, leur correspondance manifeste le sentiment des deux hommes de «converger» dans un même combat intellectuel contre les diverses variantes du scepticisme et du relativisme, alors fortement représentées dans les sciences sociales et humaines. Réagissant à l'envoi par Boudon d'un exemplaire de son Art de se persuader des idées douteuses, fragiles ou fausses (199I), Merton fait rapidement part de son enthousiasme comme de sa confiance dans l'avenir : «I haven't the least doubt that the extravagances of radical cognitive relativism are time-bound and that they will be increasingly recognized for the self-deceptive and self-destructive opinions that they are. But it may speed up the process among our faddish tribe of sociologists to have calm analyses such as yours [...]. That we are on the same wave length of course needs little further demonstration » (Lettre du 2 mars 199I). Près de trente ans plus tard, alors que l'on ne peut que constater le reflux des diverses variantes du relativisme, il apparaît que l'optimisme confiant de Merton relevait bel et bien de la fameuse catégorie dite des prédictions créatrices.

\section{CONCLUSION}

Cet article est un premier exercice d'exploitation du fonds d'archives Raymond Boudon à partir du cas particulier de sa correspondance avec Lazarsfeld et. Merton. La correspondance d'«amitiés intellectuelles » entre les trois hommes ne représente, il faut le souligner, qu'un sous-ensemble d'une correspondance plus générale dans laquelle on dénombre, pour la période I96I-200I, près de 900 correspondants. Il est l'occasion d'éclairer sur la base d'un matériau original la variété des interactions à l'œuvre entre les milieux académiques en France et aux États-Unis. Il montre de façon plus particulière de quelle manière, par-delà leur relation initiale d’apprenti à mentor, Boudon et Lazarsfeld ont joué l'un pour l'autre le rôle de médiateur au regard de leurs communautés nationales 
d’appartenance. Ces médiateurs jouent un rôle crucial dans la circulation internationale des idées sociologiques. Il ne fait guère de doute de ce point de vue que l'importance de la diffusion des travaux de Boudon aux États-Unis, sa place dans les analyses citationnelles comme celle citée en introduction de cet article, tient autant à leurs qualités intrinsèques qu’à l'attention qui a été accordée à la manière de les adapter et de les intégrer au milieu académique de réception.

L'analyse de la correspondance entre Boudon, Lazarsfeld et Merton montre également de quelle manière le premier a tenté de porter en France les qualités de l'esprit scientifique pour la sociologie incarné à ses yeux par le duo de Columbia - le «Merton-Lazarsfeld spirit»: la rigueur méthodologique, le goût pour la théorisation sociologique et la volonté de définir la sociologie comme une science créatrice de connaissances générales. Décrit par le préfacier de la traduction anglaise de l'un de ses ouvrages comme un «Unfrench sociologist», Boudon a toujours dit se sentir appartenir à un milieu académique international, gardant ce faisant ses distances par rapport aux nombreux conflits idéologiques qui ont animé la sociologie française des années 1970-1980 (Boudon, 2013, p. 607). Cette correspondance montre de quelle manière l'école de Columbia a constitué de ce point de vue pour Boudon une véritable ressource, tant stratégique qu’intellectuelle.

\section{BIBLIOGRAPHIE}

BLONDIAUX L., 1990, «Paul F. Lazarsfeld (1901-1976) et Jean Stoetzel (1910-1987) et les sondages d'opinion: genèse d'un discours scientifique», Mots, 23, «Les discours des sondages d'opinion», p. 5-23.

BONCOURT Th., 2016, «La science internationale comme ressource. Genèse et développement comparés des associations européennes de sciences sociales», Revue française de sociologie, 57-3, p. 529-561.

BORZEIX A. et ROT G., 20I0, Genèse d'une discipline. Naissance d'une revue. Sociologie du travail, Presses Universitaires de Paris Ouest.

BOUDON R., 1976, «Comment on Hauser's Review of Education, Opportunity, and Social Inequality», American Journal of Sociology, 81-5, p. II75-1।87. 
-, 1996, «Pourquoi devenir sociologue? Réflexions et évocations», Revue française de science politique, 46-I, p.52-79.

-, 1998, «L'analyse empirique de l'action de Lazarsfeld et la tradition de la sociologie comprehensive», in J. Lautman et B.-P. Lécuyer (dir.), Paul Lazarsfeld (1901-1976): La Sociologie de Vienne à New York, Paris, L'Harmattan, p.363-382.

-, 2003, Y a-t il encore une sociologie? Entretiens avec Robert Leroux, Paris, Odile Jacob.

-, 2010, La Sociologie comme science, Paris, La Découverte.

-, 2013, «Ma traversée dans le monde scientifique», I et 2, avec Brigitte Masson, Commentaire, 142-143, p.343-348 et p.603-612.

CHAUBET F., 20l4, «Faire l'histoire des sciences sociales: le cas de la sociologie française», Histoire@Politique, 22-1, p. 251-268.

-, 2013, «Michel Crozier, entre la France et les États-Unis», Vingtième siècle. Revue d'histoire, 3-119, p. 71-84.

DROUARD A., 1982, «Réflexions sur une chronologie: le développement des sciences sociales en France de 1945 à la fin des années soixante», Revue française de sociologie, 23-I, p.55-85.

DUBOIS M., 2014, «"Private knowledge" et "programme disciplinaire" en sciences sociales: étude de cas à partir de la correspondance de Robert K. Merton », L'Année sociologique, 64-I, p.79-119.

-, 2014a, «From Discovery to Invention. Sociological Study of Academic Correspondence», Revue européenne des sciences sociales, 52-2, p.7-42.

GEMELLI G., 1998, «Lazarsfeld et la France au milieu des années soixante», in J. Lautman et B.-P. Lécuyer (dir.), Paul Lazarsfeld (1901-1976): La Sociologie de Vienne à New York, Paris, L'Harmattan, p.465-50।.

HAUSER R. M., 1976, «On Boudon's Model of Social Mobility», compte rendu de R. Boudon, Education, Opportunity, and Social Inequality, American Journal of Sociology, 8I-4, p. 9II-928.

HEILBRON J., 2015, French sociology, Cornell University Press.

LANGLOIS S., 2008, «Trente ans de sociologie en France», Commentaire, I21, p. 349-359. 
LAZARSFELD P.F., 1970, Philosophie des sciences sociales, éd. par R. Boudon, Paris, Gallimard.

-, 1993, On Social Research and Its Language, éd. par R. Boudon, Chigago, Chigago University Press

LÉCUYER B.P., 2002, «Une grande figure francophile de la sociologie empirique, quantitative et mathématique et de son étude historique: Paul Lazarsfeld (190|-1976)», Mathématiques et sciences humaines, 157, p.49-104.

MARCEL J.-Ch., 200I, «Georges Gurvitch: les raisons d'un succès», Cahiers internationaux de sociologie, I10-1, p.97-119.

-, 2002, «Le premier sondage d'opinion», Revue d'histoire des sciences humaines, I-6, p. $145-153$.

-, 2004, «Une réception de la sociologie américaine en France», Revue d'histoire des sciences humaines, II-2, p. 45-68.

MENDRAS H., 1995, Comment devenir sociologue? Souvenirs d'un vieux mandarin, Arles, Actes Sud.

MIEVILLE A. et BUSINO G., 1996, «Pour une histoire de la sociologie française. Notes bibliographiques», Revue européenne des sciences sociales, 34-103, p.355-37I.

OLLION É. et ANDREW A., 2016, «French Connections: the Reception of French Sociologists in the USA (1970-2012)», European Journal of Sociology, 57-2, p. 33I-372.

POLLAK M., 1976, «La planification des sciences sociales», Actes de la recherche en sciences sociales, 2-3, p. 105-121

-, 1979, «Paul F. Lazarsfeld, fondateur d'une multinationale scientifique», Actes de la recherche en sciences sociales, 35, p. 45-59.

SÈNE P.-B., 2014, Inventaire du fonds de Raymond Boudon, Paris, Service archives de l'École des hautes études en sciences sociales.

STANKIEWICZ G., 20I8, «"Comment en finir avec une tradition dominante?": rupture et continuité dans la trajectoire de Jean Stoetzel», Revue d'histoire des sciences humaines, 18, p. 137-158.

TOPALOV Ch., 2005, «Un savant voyage: les "Lettres des États-Unis" de Maurice Halbwachs (septembre-décembre 1930) », Genèses, 58, p. I3I-I50. 